Research Paper

\title{
Lovastatin Inhibits Cancer Stem Cells and Sensitizes to Chemo- and Photodynamic Therapy in Nasopharyngeal Carcinoma
}

\author{
Yikun Peng1, Guangchun $\mathrm{He}^{2}$, Da Tang3, Li Xiong3, Yu Wen³, Xiongying Miao ${ }^{3}$, Zhangyong Hong4, Hui \\ $\mathrm{Yao}^{2}$, Chao Chen², Shichao Yan², Lu Lu², Yingke Yang5, Qinglong $\mathrm{Li}^{3 凶}$, Xiyun Deng ${ }^{2 凶}$ \\ 1. Department of Otorhinolaryngology-Head and Neck Surgery, Guizhou Provincial People's Hospital, Guiyang, Guizhou, China; \\ 2. Medical College, Hunan Normal University, Changsha, Hunan, China; \\ 3. Department of General Surgery, Second Xiangya Hospital, Central South University, Changsha, China; \\ 4. State Key Laboratory of Medicinal Chemical Biology, College of Life Sciences, Nankai University, Tianjin, China; \\ 5. College of Biology, Hunan University, Changsha, Hunan 410082, China. \\ $\square$ Corresponding authors: Qinglong Li, E-mail: 13787782059@163.com; Tel: +86-13787782059; Xiyun Deng, E-mail: dengxiyunmed@hunnu.edu.cn; Tel: \\ $+86-731-88912426$ \\ ( $)$ Ivyspring International Publisher. This is an open access article distributed under the terms of the Creative Commons Attribution (CC BY-NC) license \\ (https://creativecommons.org/licenses/by-nc/4.0/). See http://ivyspring.com/terms for full terms and conditions.
}

Received: 2017.01.07; Accepted: 2017.03.31; Published: 2017.06.03

\begin{abstract}
Nasopharyngeal carcinoma (NPC) is an Epstein-Barr virus-associated malignancy occurring at high incidence in Southeast Asia and southern China. In spite of the good response to radio- and chemo-therapy at the early stage, resistance and recurrence develop in NPC patients in the advanced setting. Cancer stem cells (CSCs) play an important role in drug resistance and cancer recurrence. Here we report that lovastatin, a natural compound and a lipophilic statin that has already been used in the clinic to treat hypercholesterolemia, inhibited the CSC properties and induced apoptosis and cell cycle arrest in sphere-forming cells derived from the 5-8F and 6-10B NPC cell lines. Furthermore, lovastatin conferred enhanced sensitivity to the chemotherapeutic and photodynamic agents in NPC CSCs. Together our findings suggest that targeting CSCs by lovastatin in combination with routine chemotherapeutic drugs or photodynamic therapy might be a promising approach to the treatment of NPC.
\end{abstract}

Key words: Lovastatin; Cancer stem cells; Nasopharyngeal carcinoma; Chemotherapy; Photodynamic therapy.

\section{Introduction}

Nasopharyngeal carcinoma (NPC) is the most common head and neck cancer in Southeast Asia and southern China [1, 2]. NPC is distinct from other head and neck malignancies in that NPC displays the characteristics of poor differentiation and strong propensity to metastasize to the regional lymph node and/or distant organs [3,4]. Synergetic actions of genetic alterations, Epstein-Barr virus (EBV) infection, and environmental factors contribute to the initiation and progression of NPC $[2,5]$. While high-dose radiation remains the primary treatment for most NPC patients, the addition of chemotherapy results in increased overall and disease-free survival especially for the advanced-stage patients [6]. Limitations in these therapeutic applications, however, exist due to undesirable side effects, local recurrence, and metastasis [7]. These limitations call for novel targets and optional approaches for the treatment of NPC.

Cancer stem cells (CSCs) are a small subset of cancer cells which share several common features such as self-renewal, multi-lineage differentiation, sphere formation, and tumorigenicity [8]. While conventional therapies are able to target the bulk tumor cells, CSCs are resistant to many current radioand/or chemo-therapeutic modalities, which might be the major reason of tumor recurrence and relapse $[9,10]$. Therefore, development of therapeutic strategies that preferentially target CSCs is of great 
importance in reducing the risk of relapse and improving the outcome of malignancies. Novel therapeutic agents that selectively target CSCs are being explored and some of them have shown promising results in preclinical and clinical studies [9, 11].

Recently, natural compounds have gained special attention in cancer therapeutics because of their ability to eliminate CSCs. Plant-derived compounds that are known to target CSCs include curcumin, sulforaphane, resveratrol, genistein, and polyphenols [12, 13]. Lovastatin (LV), also known as Monacolin $\mathrm{K}$ or mevinolin, is a natural compound derived from Monascus or Aspergillus-fermented rice and Dioscorea [14]. The content of LV is also high in the fungus Pleurotus ostreatus, Oyster mushroom [15]. LV is the first statin to be approved by the US FDA in 1987 as a cholesterol-lowering drug. Statins are 3-hydroxy-3-methylglutaryl coenzyme A (HMG-CoA) reductase inhibitors that are widely used in the clinic for the treatment of hypercholesterolemia, thus reducing the incidence of cardiovascular and cerebrovascular events [16]. Anti-cancer effects of stains have been reported in a variety of cancer types including breast cancer [17, 18], prostate cancer [19], esophageal carcinoma [20], and ovarian cancer [21]. Recently, it has been shown that statins such as simvastatin target CSCs derived from breast cancer [18]. However, it is not known whether statins have similar effects on NPC CSCs. In the present study, we have provided evidence to demonstrate that $\mathrm{LV}$, one of the naturally occurring lipophilic statins, has the ability to inhibit proliferation and self-renewal and induce apoptosis and cell cycle arrest of NPC CSCs. We also showed that LV could synergistically enhance the sensitivity of NPC CSCs to chemotherapy and photodynamic therapy. These observations will open the window to repurpose the lipid-lowering drugs as anti-cancer therapeutics for the treatment of NPC.

\section{Materials and Methods}

\section{Reagents}

Antibodies against CD44 (Abcam, Cat \#ab51037), c-Myc (Abcam, Cat \#ab32072), CBFb (Abcam, Cat \#ab133600), and GAPDH (ZSGB-BIO, Cat \#TA-08) were obtained commercially. FITC-conjugated CD44 antibody (BD, Cat \#560977) was purchased from BD Biosciences (Franklin Lakes, NJ, USA). Statins were purchased from Abcam (atorvastatin [ab145604], lovastatin [ab120614], and simvastatin [ab120505]) or Sigma-Aldrich (St Louis, MO, USA) and dissolved in DMSO as a $20 \sim 30 \mathrm{mM}$ stock solution and stored at $-20^{\circ} \mathrm{C}$ before use.
Chemotherapeutic drugs (cyclophosphamide [CPA], cisplatin [DDP], doxorubicin [DOX], and paclitaxel [TXL] were obtained from the in-hospital pharmacy or Selleck (Shanghai, China). The photosensitizer polyhematoporphyrin $\left(\mathrm{C}_{34} \mathrm{H}_{38} \mathrm{~N}_{4} \mathrm{NaO}_{5}\right.$, Photosan-II [PS-II]) was purchased from Seehof Laboratorium F\&E GmbH (Wesselburenerkoog, Germany).

\section{Cell culture and treatments}

NPC cell lines 5-8F and 6-10B were obtained from Cancer Research Institute of Central South University. Both cell lines were maintained in RPMI 1640 medium supplemented with $10 \%$ fetal bovine serum (FBS) at $37^{\circ} \mathrm{C}$ in a humidified atmosphere of $5 \%$ $\mathrm{CO}_{2}$ as described [22]. The cells were tested for mycoplasma contamination and rendered mycoplasma-negative before the experiments.

For treatment, the cells were seeded in culture dishes or plates $\left(1 \times 10^{5}\right.$ cells $\left./ \mathrm{ml}\right)$ and allowed to grow overnight under normoxia $\left(21 \% \mathrm{O}_{2}\right)$ before treatment. The next day, the respective statin with or without the chemotherapeutic or photodynamic drug was added to the cells at various concentrations and the culture was continued for the desired period of time under hypoxia $\left(1 \% \mathrm{O}_{2}\right)$. Hypoxic culture condition was achieved in a hypoxic chamber (Thermo Fisher Scientific, Waltham, MA, USA) by inflation of $\mathrm{N}_{2}$ into the air-filled chamber as described [17].

\section{Tumorsphere-forming culture}

The CSCs were enriched in 6-well ultra-low attachment plates $(2,500$ cells/well $)$ in serum-free medium according to the published tumorsphere culture technique [23]. The culture medium consisted of DMEM/F-12 (Life Technologies, Carlsbad, CA, USA) supplemented with $1 \times$ B27 (without vitamin A) (Life Technologies 12587-010), $20 \mathrm{ng} / \mathrm{ml}$ EGF (Prospec, East Brunswick, NJ, USA, Cyt-217-b), 20 ng/ml bFGF (Prospec Cyt-218-b), 0.4\% BSA (Sigma, St. Louis, MO, USA), $4 \mu \mathrm{g} / \mathrm{ml}$ Insulin (Genview, Beijing, China, FI174). The tumorspheres were passaged every 6 days, with the sphere-forming capacity retained for at least six generations. The CSC phenotype was characterized according to the expression of NPC CSC surface marker CD44 and the self-renewal capacity.

\section{Self-renewal assay}

Self-renewal assay was performed according to the published procedure [24] with some modifications. Briefly, single cell suspension of sphere-forming cells (SFCs) at a density of 100 cells/ml (100 $\mu \mathrm{l} /$ well) was plated in 96-well ultra-low attachment plates in sphere culture medium. The next day, different concentrations of LV and/or drugs were added to the medium and the culture was 
continued for $72 \mathrm{~h}$ under hypoxia followed by image capture (one image per well taken in the center of each well). The surface areas of tumorspheres were measured using the Adobe Photoshop CS3 software (San Jose, CA, USA). The sphere formation unit was obtained by multiplying the number of tumorspheres and the surface areas of the tumorspheres in each well.

\section{Cell proliferation/viability assay}

To assess the effects of LV on cell proliferation, NPC parental cells and CSCs were seeded in 96-well plates at a density of $1 \times 10^{5}$ cells $/ \mathrm{ml}$ (for parental cells) or $4 \times 10^{4}$ cells $/ \mathrm{ml}$ (for CSCs) in a volume of 100 $\mu \mathrm{l} /$ well. The next day, the cells were treated with LV or a combination of LV and chemotherapeutic drugs and cultured under hypoxia $\left(1 \% \mathrm{O}_{2}\right)$ for $72 \mathrm{~h}$. Cell proliferation/viability was measured using an Alamar Blue cell viability assay kit (Promega, Madison, WI, USA) as described by our group [17]. The values of half maximal inhibitory concentration $\left(\mathrm{IC}_{50}\right)$ were calculated using the GraphPad Prism software (La Jolla, CA, USA).

\section{Cell death analysis}

The cells seeded into $35-\mathrm{mm}$ dishes at a density of $4 \times 10^{4}$ cells $/ \mathrm{ml}$ were treated with different concentrations of LV for $72 \mathrm{~h}$ under hypoxia. Cell death was determined using the Annexin V-FITC/propidium iodide (PI) apoptosis detection kit (Seven Sea Biotech, Shanghai, China) with a BD FACS Canto II flow cytometer (Franklin Lakes, NJ, USA) according to the previously published method [25].

\section{Cell cycle analysis}

The cells $\left(1 \times 10^{5}\right.$ cells $\left./ \mathrm{ml}\right)$ grown in $35-\mathrm{mm}$ dishes were treated with different concentrations of $\mathrm{LV}$ for $72 \mathrm{~h}$ under hypoxia. The cells were fixed in $70 \%$ ethanol followed by PI staining of DNA and analyzed by flow cytometry as described [26].

\section{Cell lysate preparation and Western blot analysis}

Whole cell lysates were prepared from cultured cells using $1 \mathrm{X}$ cell lysis buffer (Cell Signaling Technology, Danvers, MA, USA) with 1X protease inhibitor cocktail (Complete Mini, Roche, Mannheim, Germany) and $1 \mathrm{mM}$ phenylmethanesulfonyl fluoride (Sigma-Aldrich) added. The supernatants (whole cell lysates) were collected and quantified by the BCA protein quantification method. The lysates were mixed with the LDS sample buffer and reducing agent and denatured by boiling. The same quantity of protein from each sample was then separated on $10 \%$ denaturing PAGE gels followed by incubation with the respective primary antibody and the
HRP-conjugated secondary antibody and subsequent ECL development according to our standard procedure [27].

\section{Drug interaction analysis}

Drug interaction was analyzed according to the Chou-Talalay method [28]. Briefly, the cells were treated with serial dilutions of therapeutic drugs and $L V$, either individually or in combination, under hypoxia for the desired period of time. Cell viability and self-renewal assays were performed as described above. Combination index (CI) was calculated using the CompuSyn software (New York, NY, USA). Synergistic, additive, or antagonistic combination was determined according to the calculated CI values (< $1.0,=1.0$, or $>1.0$, respectively).

\section{Photodynamic therapy}

The subconfluent cells were pretreated with LV or vehicle under hypoxia for $24 \mathrm{~h}$ followed by treatment with different concentrations of PS-II in culture medium for $4 \mathrm{~h}$. After that, the cells were exposed to a $630 \mathrm{~nm}$ laser with a light intensity of $10 \mathrm{~J} / \mathrm{cm}^{2}$ for $60 \mathrm{~s}$. The laser was emitted from a semiconductor laser device manufactured and provided by the Laser Medical Tech Co., Ltd (Shenzhen, China). The irradiated cells were further incubated for different time durations depending on the experimental purposes.

\section{Statistical analysis}

The quantitative data obtained from at least two independent experiments were presented as mean \pm SEM and analyzed by one-way ANOVA followed by Holm Sidak post hoc test. $P<0.05$ was considered statistically significant.

\section{Results}

\section{Lovastatin preferentially inhibits NPC stem cells compared with non-stem cells}

In this study, we used two NPC subclones, $5-8 \mathrm{~F}$ and $6-10 \mathrm{~B}$, that were derived from the same precursor cell line (SUNE-1) but with different metastatic potential [29] as our cell model. We enriched CSCs from both $5-8 \mathrm{~F}$ and $6-10 \mathrm{~B}$ cells using the tumorsphere culture technique as recently described [23]. These cells formed tumorspheres approximately 2 to 3 days after initial culture, which showed obvious morphological difference compared with the parental cells. Prolonged culture maintained the ability of these cells to form tumorspheres (Fig. S1). Flow cytometric analysis revealed that these SFCs had increased level of CD44, the characteristic marker phenotype of NPC CSCs [8] (Fig. S2). 
We first examined the effect of LV on the proliferation of NPC CSCs and parental cells under a hypoxic culture condition. We found that $\mathrm{LV}$ inhibited the proliferation of both parental 5-8F and 6-10B cells and the SFCs derived from them, as evaluated by Alamar blue cell viability assay (Fig. 1A) and microscopic observation (Fig. 1B). However, LV had a greater inhibitory effect on NPC SFCs, as demonstrated by significantly lower $\mathrm{IC}_{50}$ values for SFCs than their respective parental counterpart (5-8F: $4.79 \pm 1.23$ vs. $11.42 \pm 4.14 \mu \mathrm{M}, P<0.05 ; 6-10 \mathrm{~B}: 3.01 \pm$ 0.42 vs. $9.30 \pm 3.67 \mu \mathrm{M}, P<0.05)$. These results suggest that the NPC CSCs might be a valid therapeutic target of LV.
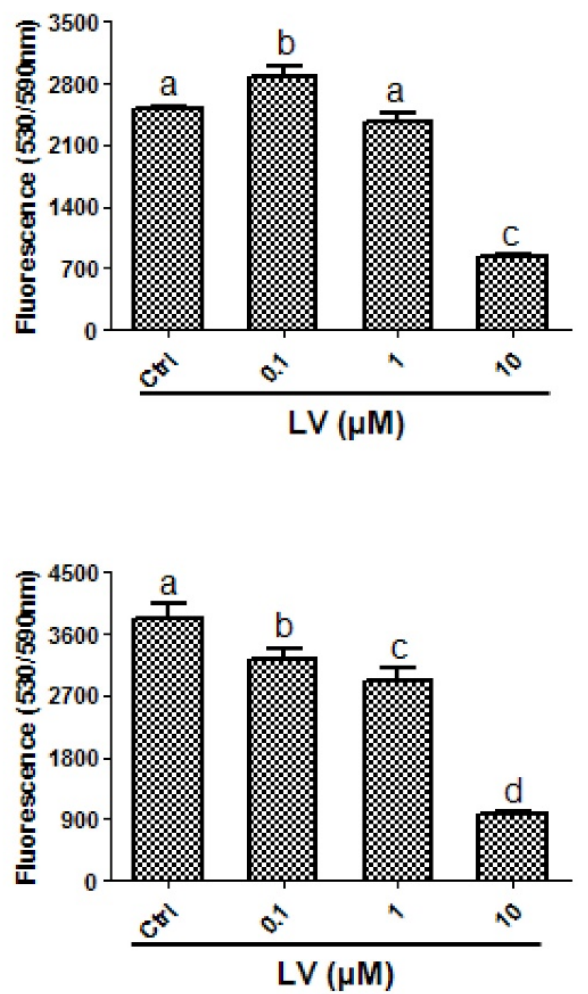

B

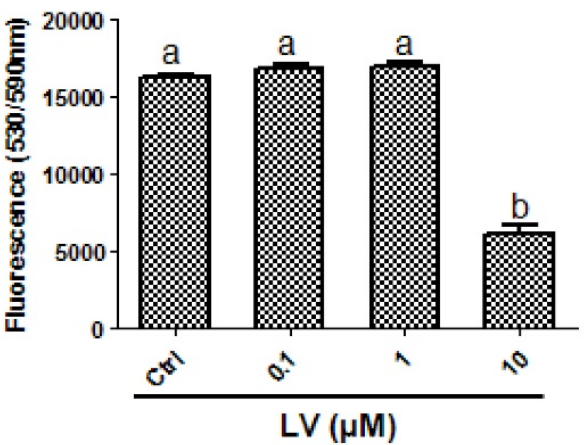

$\mathrm{LV}(\mu \mathrm{M})$

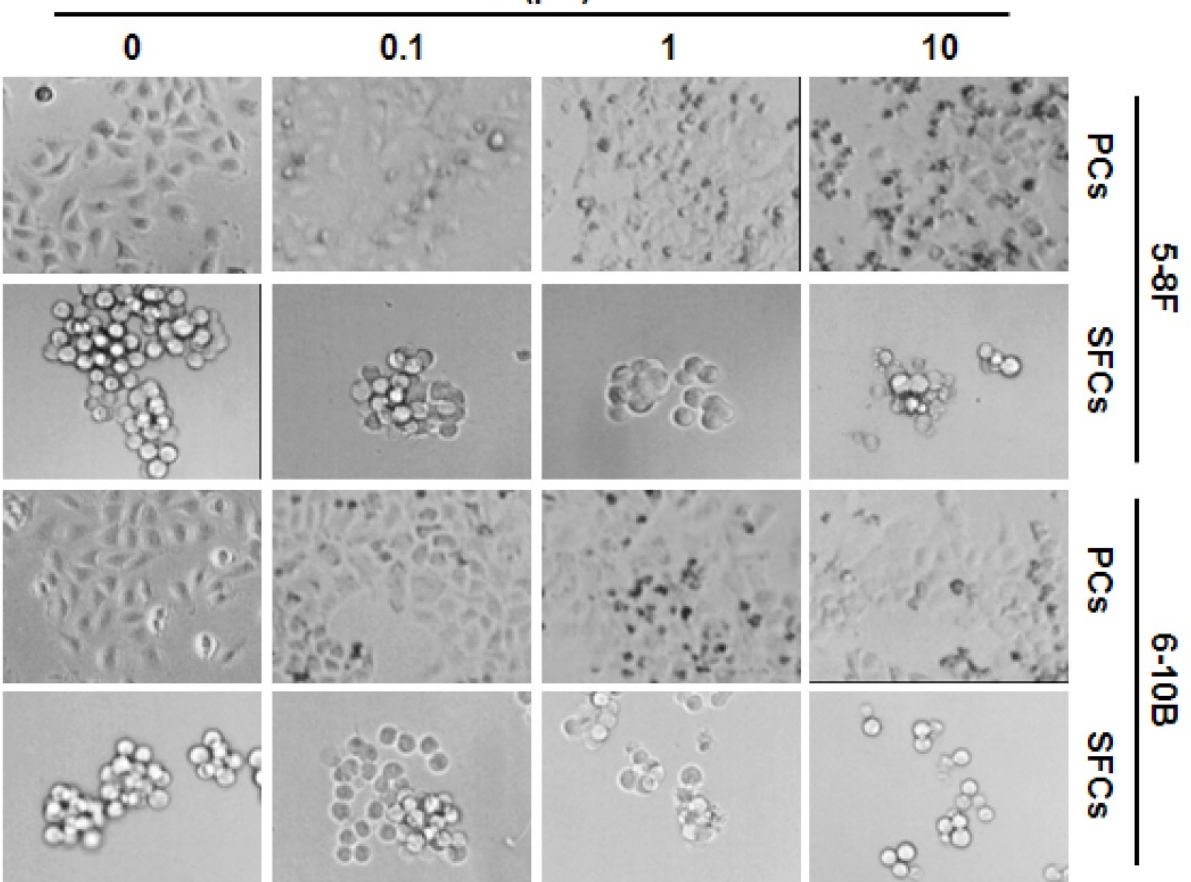

Figure 1. Lovastatin preferentially inhibits the proliferation of NPC sphere-forming cells as compared with the parental cells. (A) $5-8 \mathrm{~F}$ or 6-10B parental or sphere-forming cells were treated with different concentrations of LV under hypoxia for $72 \mathrm{~h}$ and subjected to Alamar Blue cell viability assay. (B) Photomicrographic images were taken after Alamar blue analysis. Original magnification: $\times 200$. Different lower-case letters (a, b, c) denote statistically significant difference between the groups. PCs, parental cells; SFCs, sphere-forming cells. 


\section{Lovastatin inhibits stemness properties of NPC stem cells}

The effect of LV on the self-renewal capacity of NPC CSCs was analyzed by culturing SFCs in the presence or absence of LV. We found that LV, when used at $1 \mu \mathrm{M}$ final concentration, could inhibit the self-renewal capacity of $5-8 \mathrm{~F}$ SFCs by $>70 \%$ compared with the vehicle control. We also compared the inhibitory effect of LV with the other two statins, atorvastatin and simvastatin. The three lipophilic statins performed similarly in inhibiting the self-renewal capacity of 5-8F SFCs (Fig. 2A, B).

To determine whether LV inhibits the stemness marker proteins of NPC cells, we performed Western blot analysis on NPC SFCs treated with different concentrations of LV under hypoxia. Decreased protein levels of CD44 and SOX2 (NPC CSC marker proteins [8]), c-Myc (a master regulatory gene of CSCs [30]), core binding factor $\beta(\mathrm{CBFb}$, a transcription factor required for the emergence of hematopoietic stem cells [31]), and Snail (a transcription factor involved in the induction of EMT and CSC renewal [32] were observed in 5-8F NPC SFCs treated with LV in a dose-dependent manner (Fig. 2C). These results suggest that LV and possibly other lipophilic statins could inhibit the stemness properties of NPC CSCs.

\section{Lovastatin induces apoptosis and cell cycle arrest in NPC stem cells}

The effect of LV on cell death in NPC CSCs was examined by staining with FITC-Annexin V and propidium iodide (PI) followed by flow cytometry. We found that LV dose-dependently induced apoptosis in $5-8 \mathrm{~F}$ and $6-10 \mathrm{~B}$ SFCs as shown by the increase in the fraction of cells in the early- and late-apoptosis stage. While low-dose $(1 \mu \mathrm{M})$ of $\mathrm{LV}$ could induce a near 2-fold increase in the number of apoptotic cells compared with the vehicle control in either cell type, $10 \mu \mathrm{M}$ of LV led to a 3.80 -fold or 4.95 -fold increase in the proportion of apoptotic cells in 5-8F or 6-10B SFCs, respectively (Fig. 3).

\section{A}
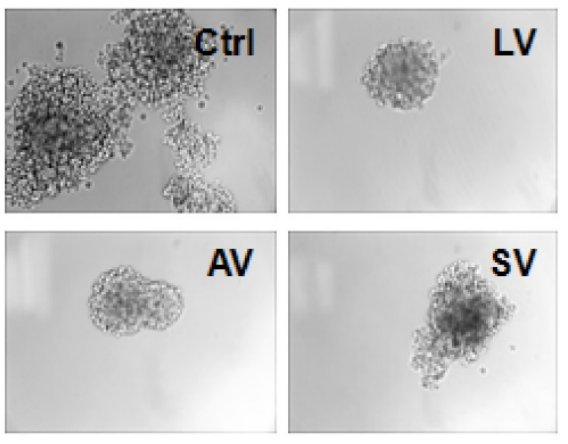
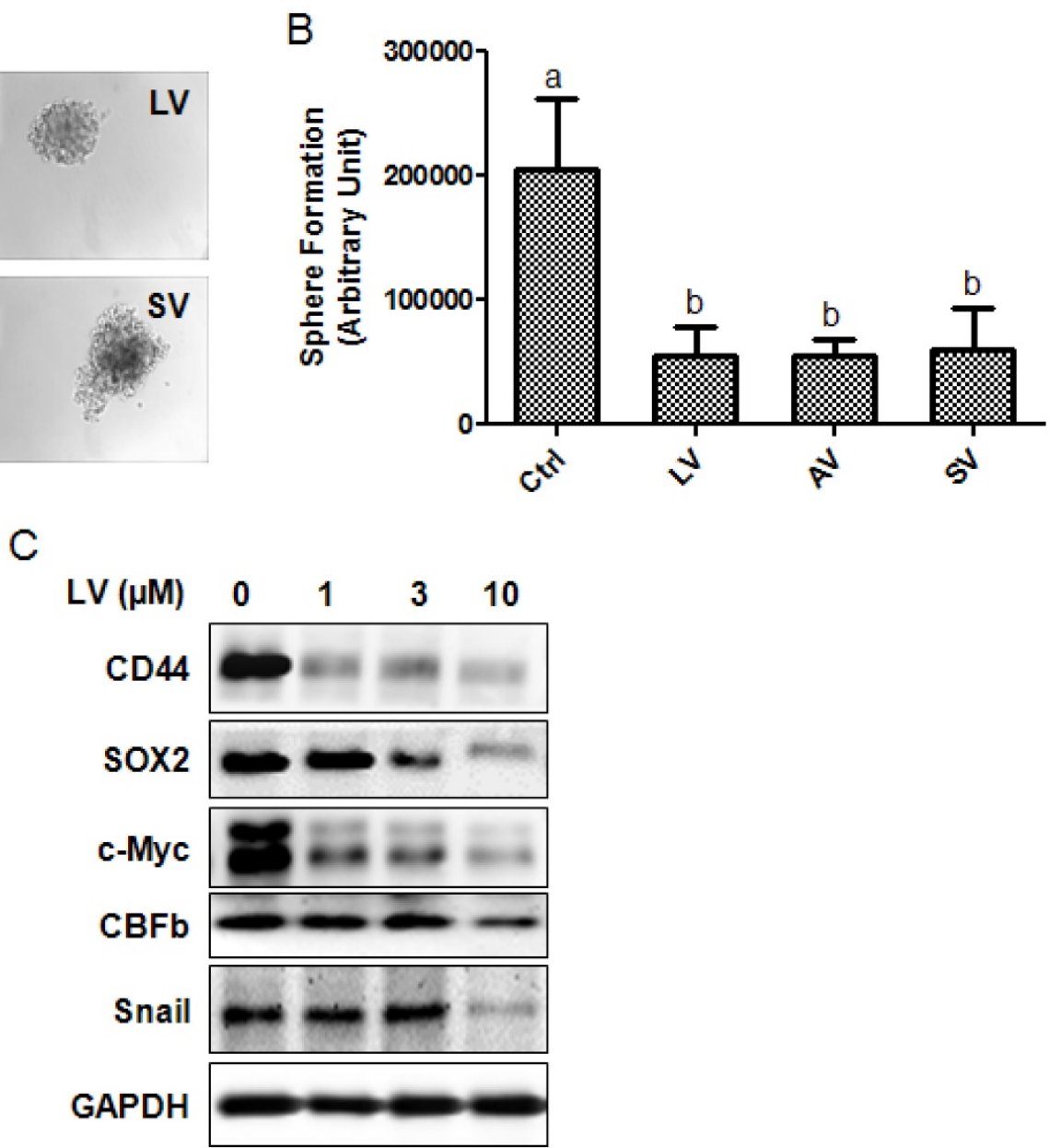

Figure 2. Statins inhibit the stemness properties of NPC sphere-forming cells. (A, B) NPC sphere-forming cells were cultured in ultra-low attachment plates in the presence of different statins $(1 \mu \mathrm{M})$ in stem cell culture medium. (A) Photomicrographic images were taken at the end of the 72 -h culture period. Original magnification: $\times 200$. (B) Sphere formation was quantified by multiplying the number of tumorspheres and the surface areas of the tumorspheres in each well. (C) The sphere-forming cells were treated with different concentrations of LV for $48 \mathrm{~h}$ and subjected to Western blot analysis. LV, lovastatin; AV, atorvastatin; SV, simvastatin. 
Induction of cell cycle entry is important in waking up the quiescent CSCs in the tumor mass [9]. We tested whether LV could impact the cell cycle progression in NPC CSCs using the standard PI staining-flow cytometry technique. Exposure of 5-8F and 6-10B SFCs to LV resulted in cell cycle arrest at the $\mathrm{G}_{2} / \mathrm{M}$ phase, while decreasing the number of cells in the $G_{1}$ and/or $S$ phase (Fig. 4). These results indicate that LV may block the NPC CSCs at the $\mathrm{G}_{2} / \mathrm{M}$ checkpoint, preventing cells from entering mitosis and thus stopping proliferation.

\section{LV increases the sensitivity of NPC stem cells to chemotherapeutic agents and photodynamic therapy}

CSCs are the major reason of the failure of chemotherapy [33]. Therefore, we tested whether LV could sensitize NPC CSCs to chemotherapeutic drugs routinely used in the clinic to treat NPC, including CPA, DDP, DOX, and TXL. As shown in Fig. 5A, in the absence of $L V$, these drugs had very little, if any, inhibitory effect on 5-8F or 6-10B SFCs in their respective clinically relevant dose range. However, LV synergistically enhanced the sensitivity of $5-8 \mathrm{~F}$ and 6-10B SFCs to CPA. LV did not show synergistic effect with the other three chemotherapeutic drugs, i.e., DDP, DOX, or TXL within the drug concentration range examined (data not shown). In addition, LV also synergistically enhanced the effect of CPA on inhibiting the self-renewal capacity of 5-8F SFCs (Fig. $5 B, C)$. These results suggested that $L V$ could sensitize the NPC CSCs to the chemotherapeutic drug CPA.

A

$\operatorname{LV}(\mu \mathrm{M})$
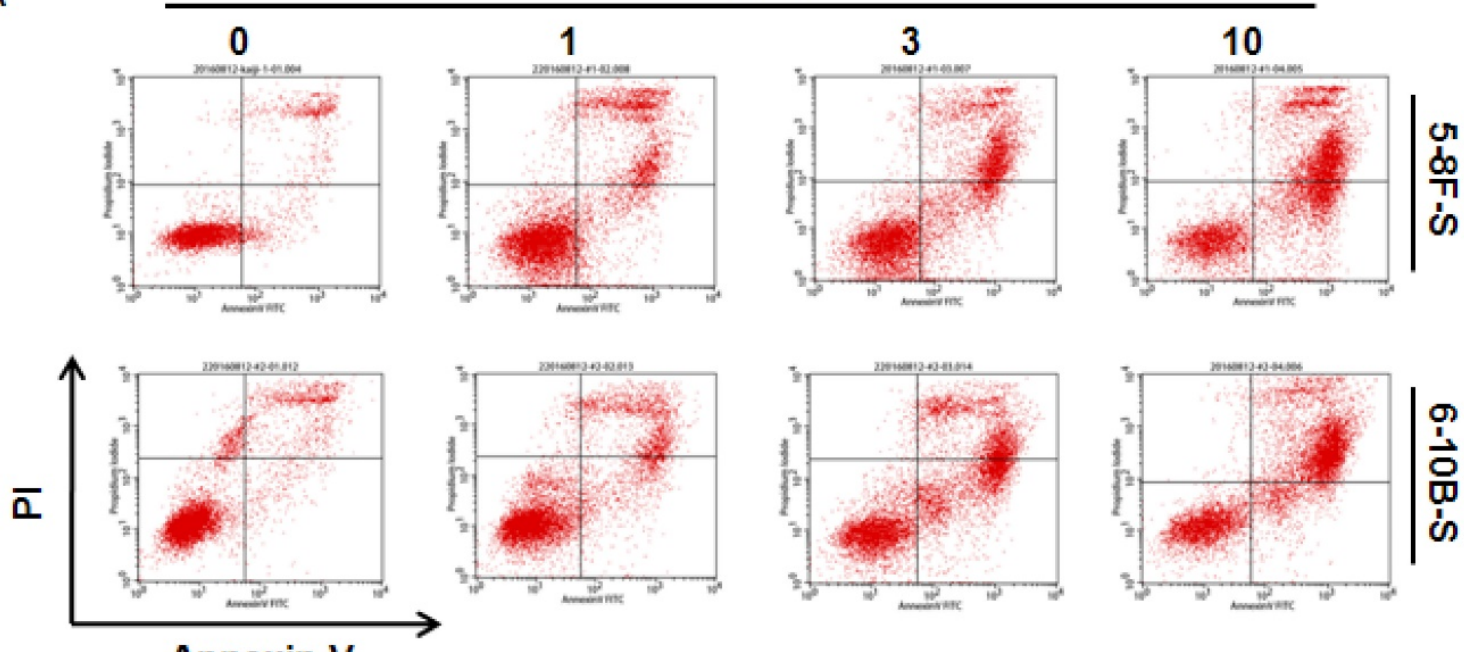

Annexin V

B $\quad 5-8 \mathrm{~F}-\mathrm{S}$

\begin{tabular}{|c|c|c|c|c|}
\hline & \multicolumn{4}{|c|}{ LV $(\boldsymbol{\mu M})$} \\
\hline Cell Fraction & $\mathbf{0}$ & $\mathbf{1}$ & $\mathbf{3}$ & $\mathbf{1 0}$ \\
\hline Early apoptosis (Q4) & $6.96 \%$ & $9.58 \%$ & $18.07 \%$ & $27.37 \%$ \\
\hline Late apoptosis/necrosis (Q2) & $11.57 \%$ & $24.73 \%$ & $30.6 \%$ & $43.04 \%$ \\
\hline Necrosis only (Q1) & $0.89 \%$ & $1.43 \%$ & $1.09 \%$ & $0.8 \%$ \\
\hline Non-apoptosis (Q3) & $80.58 \%$ & $64.26 \%$ & $50.24 \%$ & $28.78 \%$ \\
\hline
\end{tabular}

6-10B-S

\begin{tabular}{|c|c|c|c|c|}
\hline & \multicolumn{4}{|c|}{ LV $(\boldsymbol{\mu}$ M) } \\
\hline Cell Fraction & $\mathbf{0}$ & $\mathbf{1}$ & $\mathbf{3}$ & $\mathbf{1 0}$ \\
\hline Early apoptosis (Q4) & $2.64 \%$ & $10.02 \%$ & $29.22 \%$ & $10.7 \%$ \\
\hline Late apoptosis/necrosis (Q2) & $10.56 \%$ & $17.14 \%$ & $25.44 \%$ & $54.7 \%$ \\
\hline Necrosis only (Q1) & $4.15 \%$ & $1.46 \%$ & $0.86 \%$ & $0.86 \%$ \\
\hline Non-apoptosis (Q3) & $82.66 \%$ & $71.38 \%$ & $44.48 \%$ & $33.74 \%$ \\
\hline
\end{tabular}

Figure 3. Lovastatin promotes apoptosis in NPC sphere-forming cells. (A) NPC sphere-forming cells were treated with various concentrations of LV for 72 $\mathrm{h}$ under hypoxia and subjected to Annexin V/PI staining and flow cytometry. (B) Summary of the percentages of the number of cells in each fraction of cell death/survival. 


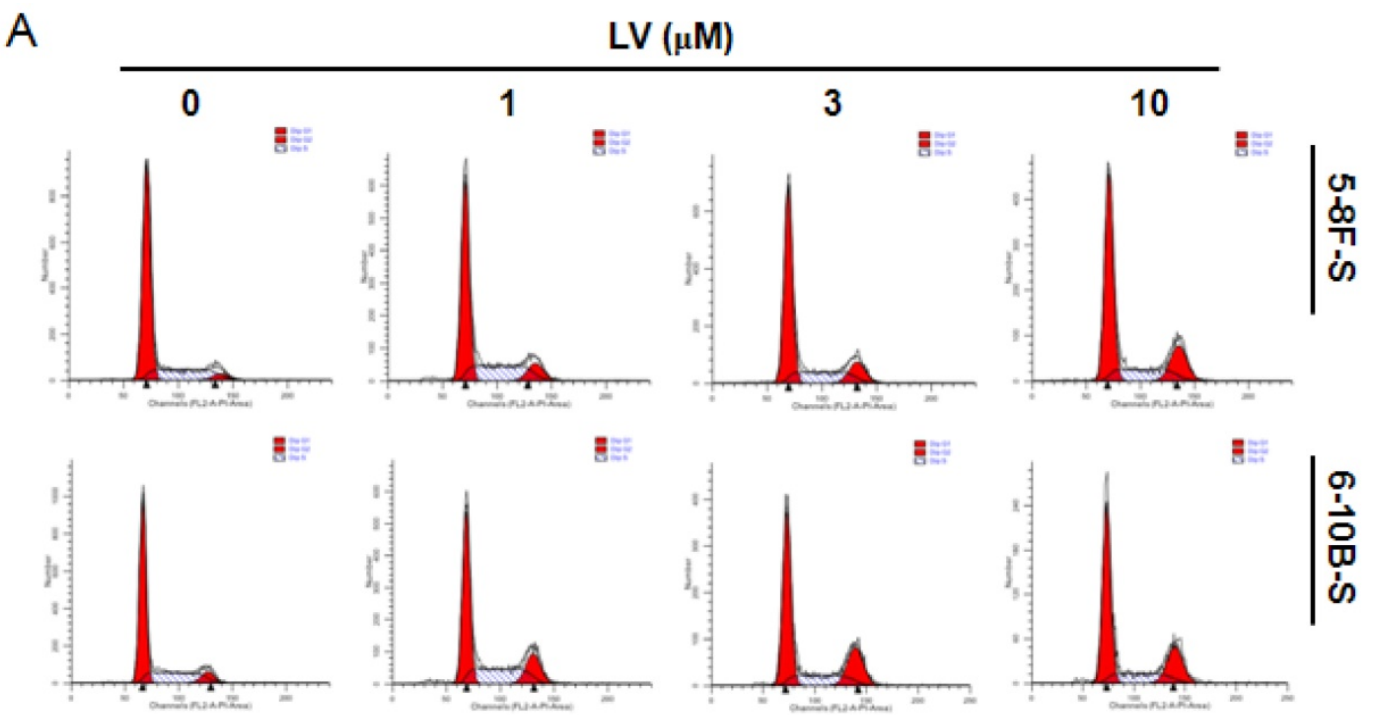

B

5-8F-S

Cell Cycle Phase

$\mathrm{G}_{1}$

$S$

$\mathrm{G}_{2} / \mathrm{M}$

6-10B-S

Cell Cycle Phase

$\mathrm{G}_{1}$

$S$

$\mathrm{G}_{2} / \mathrm{M}$

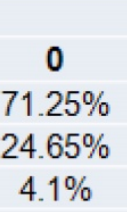

$4.1 \%$

$\mathbf{1}$ LV $(\boldsymbol{\mu})$
$\mathbf{1}$
$59.85 \%$
$30.21 \%$
$9.95 \%$

\section{$(\mu \mathrm{M})$}

3

$63.54 \%$

$23.27 \%$

$13.19 \%$
10

$60.03 \%$

$20.69 \%$

$19.28 \%$

Figure 4. Lovastatin induces cell cycle arrest in NPC sphere-forming cells. (A) NPC sphere-forming cells were treated with various concentrations of LV for $72 \mathrm{~h}$ under hypoxia and subjected to PI staining and flow cytometry. (B) Summary of the percentages of the number of cells in each phase of the cell cycle.

In a similar way, we investigated whether LV could enhance the sensitivity of NPC SFCs to PDT. As depicted in Fig. 6A, photodynamic treatment with PS-II, when used alone, did not significantly decrease cell viability in either 5-8F or 6-10B SFCs. However, when combined with LV, laser-activated PS-II dose-dependently decreased cell viability in both 5-8F and 6-10B SFCs. Similar results were obtained for apoptosis induction in both $5-8 \mathrm{~F}$ and 6-10B SFCs when PS-II was combined with LV (Fig. 6B). These results suggested that $\mathrm{LV}$ could sensitize the CSC population of NPC to PDT.

\section{Discussion}

In the present study, we show that LV, a lipophilic statin already used in the clinic to treat cardiovascular disease, inhibited the stemness properties and induced apoptosis and cell cycle arrest of CSCs derived from two NPC cell lines, 5-8F and
6-10B. In addition, we found that LV conferred enhanced sensitivity of NPC CSCs to chemotherapeutic and photodynamic therapeutic agents. The existing treatment modalities usually fail to cure cancer because they cannot completely eradicate CSCs [34]. Therefore, agents that preferentially target CSCs will have a great impact on disease progression and are clinically urgently needed. In our study, although LV inhibited the proliferation of both NPC parental cells and CSCs, the effect on CSCs was much more potent than on parental cells $\left(\mathrm{IC}_{50}\right.$ : 4.79 vs. $11.42 \mu \mathrm{M}$ for $5-8 \mathrm{~F} ; 3.01$ vs. $9.30 \mu \mathrm{M}$ for $6-10 \mathrm{~B})$. These observations are in line with a previous report that the growth inhibitory activity of statins is more potent in mesenchymal-like cells with higher protein synthesis rate [35]. The $\mathrm{IC}_{50}$ doses for LV are achievable in clinical trials for patients with various types of tumors in which serum levels of LV reached up to $3.9 \mu \mathrm{M}$ when taken at $4 \mathrm{mg} / \mathrm{kg}$ dose level [36]. In this sense, statins may represent a novel 
class of drugs that have the potential of targeting the CSCs, i.e., the refractory cells, in NPC.

Accumulating evidence has established that the CSCs are responsible for drug resistance and recurrence in cancer. Indeed, the CSC population is more resistant to conventional cancer therapeutics than the non-CSC population [11]. Therefore, sensitizing CSCs to chemotherapy holds great promise in cancer treatment. Cyclophosphamide (CPA), an alkylating agent that belongs to the group of oxazaphosporines, has been used in local-regional NPC as an adjuvant chemotherapeutic agent after radiotherapy [37]. One of the problems of CPA use in the clinic is that besides its anti-mitotic and anti-replicative effects, CPA also has immunosuppressive properties [38]. We found that in the presence of LV, the NPC CSCs responded to CPA more robustly than using $\mathrm{CPA}$ alone, suggesting that LV could confer enhanced sensitivity to CPA in NPC CSCs through inhibiting the stemness properties.

PDT is a photochemistry-based therapeutic modality in which a photosensitizer is energized by light to produce photodamage of the target cells. PDT
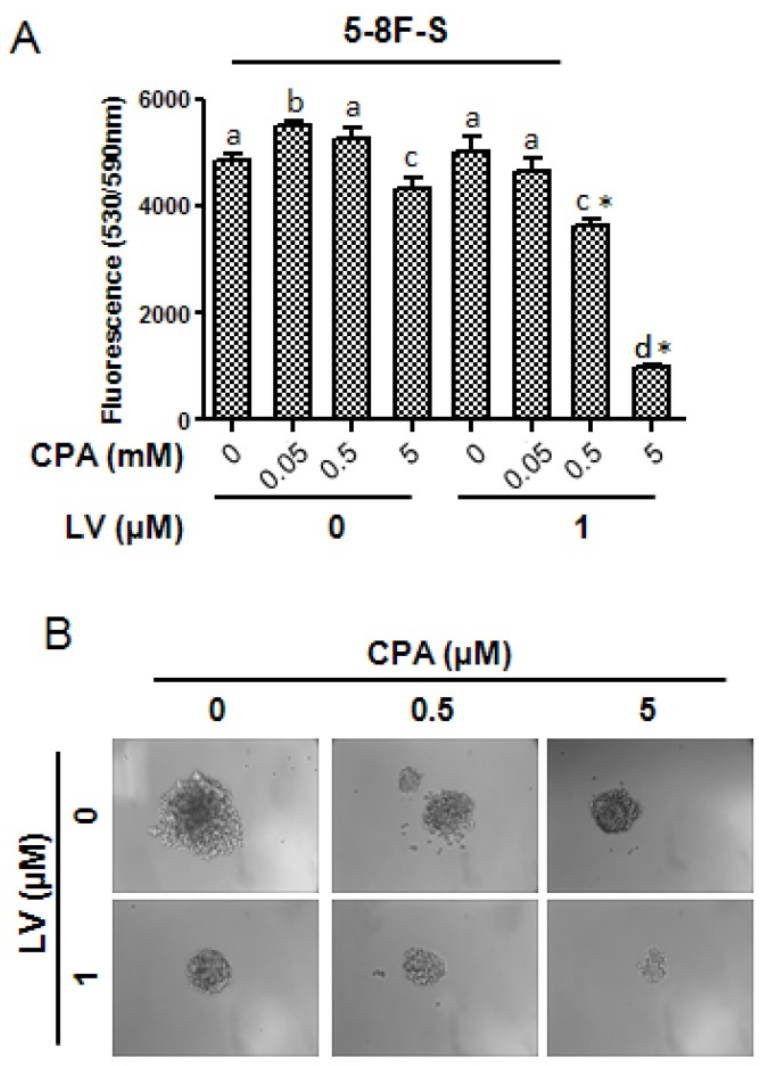

is a clinically approved, minimally invasive therapeutic procedure that can exert selective cytotoxicity toward cancer cells [39]. The mechanisms underlying PDT's anti-cancer efficacy may include anti-drug resistance effect, anti-tumor immunity, and photodamage of the tumor microenvironment [40]. PDT has emerged as a promising novel treatment for drug-resistant CSCs because of the selectivity of the photosensitizers [41]. Herein, we showed that the NPC CSCs were resistant to the $1^{\text {st }}$ generation photosensitizer PS-II in cell culture. However, when the cells were pretreated with LV, PDT using PS-II exhibited cytotoxicity in NPC CSCs. Since PDT offers the advantage of dual selectivity, i.e., both the photosensitizer and the light are required for efficient photodamage, the issue of non-selective cytotoxicity can be avoided or minimized. The above observations together with the reports in the literature support the use of statins as adjuvant therapeutic agents in combination with currently available chemotherapeutic drugs or PDT in the treatment of NPC, pending more in vivo investigations and clinical trials in the future.
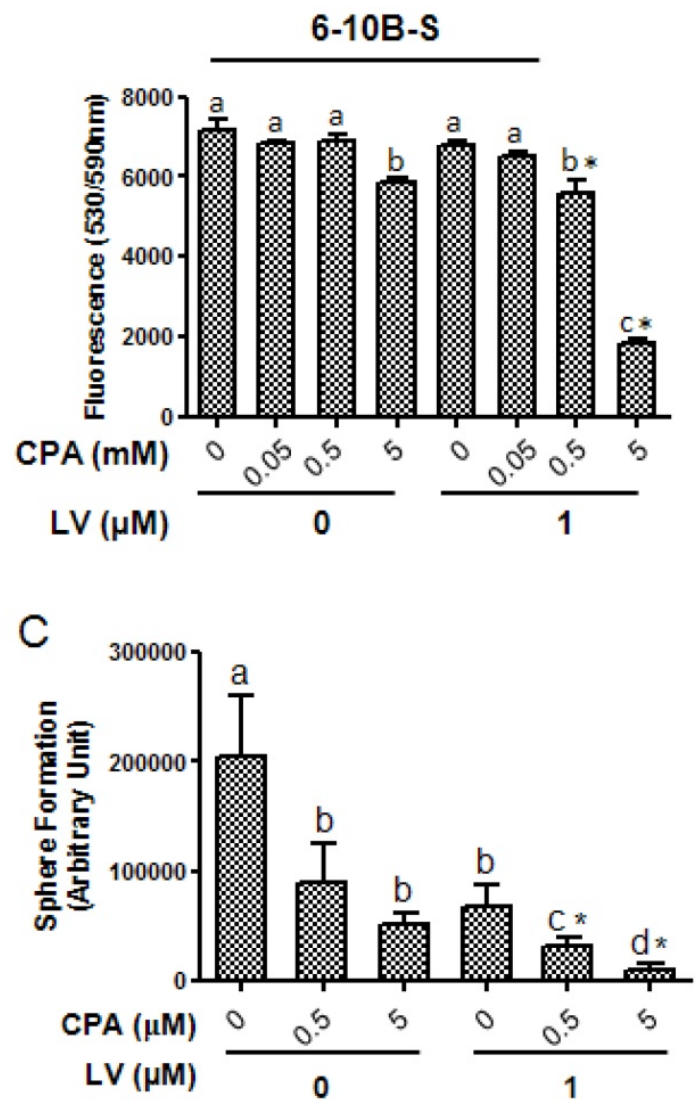

Figure 5. Lovastatin confers enhanced sensitivity of NPC sphere-forming cells to cyclophosphamide. NPC sphere-forming cells were treated with various concentrations of chemotherapeutic drugs in the presence or absence of LV under hypoxia. (A) Alamar blue cell viability assay was performed $48 \mathrm{~h}$ after drug treatment. (B) Photomicrographic images were taken at the end of the 72 -h culture period. Original magnification: $\times 200$. (C) Sphere formation was quantified by multiplying the number of tumorspheres and the surface areas of the tumorspheres in each well. Different lower-case letters (a, b, c) denote statistically significant difference between the groups. Combination index $(\mathrm{Cl})$ was calculated using the CompuSyn software. * indicates $\mathrm{Cl}<1.0$. 
A

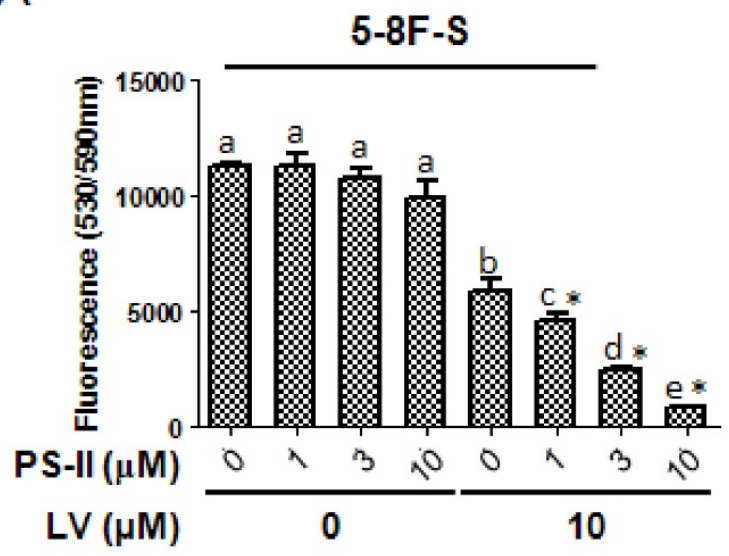

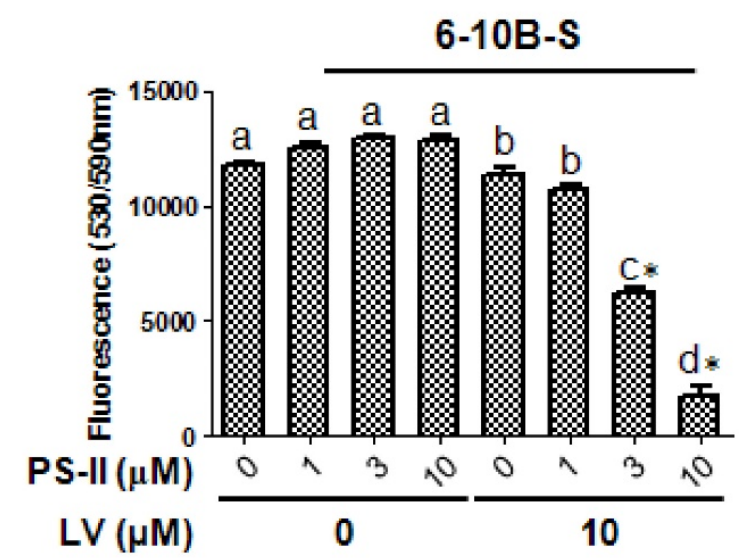

B

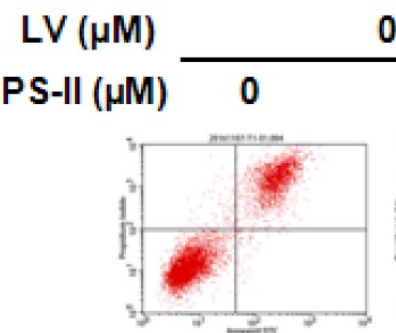

0

3
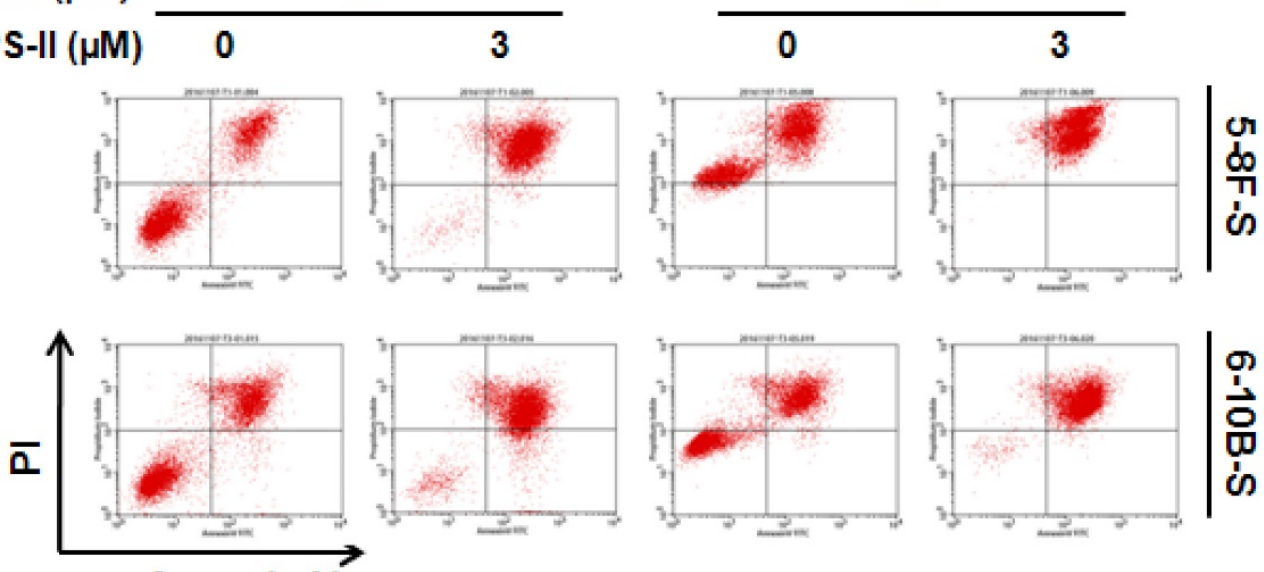

10
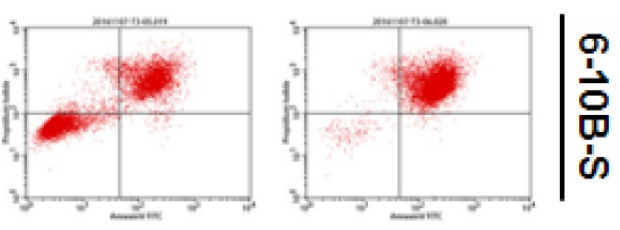

Annexin V

\section{5-8F-S}

Figure 6. Lovastatin confers enhanced sensitivity of NPC sphere-forming cells to photodynamic therapy. NPC sphere-forming cells were treated with various concentrations of PS-II with or without LV pretreatment under hypoxia. (A) $48 \mathrm{~h}$ after photodynamic treatment, the cells were subjected to Alamar blue cell viability assay. (B) $72 \mathrm{~h}$ after photodynamic treatment, the cells were subjected to Annexin V/PI staining and flow cytometry. Different lower-case letters (a, b, c) denote statistically significant difference between the groups. Combination index $(\mathrm{Cl})$ was calculated using the CompuSyn software. * indicates $\mathrm{Cl}<1.0$.

In summary, our studies provide the evidence, for the first time, that LV has the potential to selectively target the CSCs and confer enhanced sensitivity to chemotherapeutic and photodynamic therapeutic agents in NPC. Since the tolerability and pharmacology of LV and other lipophilic statins are well defined, they can enter clinical trials rapidly either as single agents or as adjuvants. These clinical trials of LV in NPC, preferably as adjuvant therapeutic agent, can thus be considered upon the completion of the evaluation in more clinically relevant models such as the patient-derived xenograft (PDX) model [42]. Since the bioavailability of LV is still low after oral administration [43], other formulations (such as nanoparticle delivery systems) that can increase the bioavailability and stability and that can be directly administered through i.v. injection need to be investigated.

\section{Abbreviations}

CI: Combination index

CPA: Cyclophosphamide

CSC: Cancer stem cell

DDP: Cis-diamminedichloridoplatinum (II)

EBV: Epstein-Barr virus

$\mathrm{IC}_{50}$ : Half maximal inhibitory concentration

LV: Lovastatin

NPC: Nasopharyngeal carcinoma

PCs: Parental cells

PDT: Photodynamic therapy

PS-II: Photosan-II

SFCs: Sphere-forming cells 


\section{Supplementary Material}

Supplementary figures.

http://www.jcancer.org/v08p1655s1.pdf

\section{Acknowledgements}

The authors would like to thank the colleagues at Cancer Research Institute of Xiangya Medical College, Central South University for the generous help in providing the cell lines and the protocol for tumorsphere culture of NPC.

\section{Competing Interests}

The authors have declared that no competing interest exists.

\section{References}

[1] Chang ET, Adami HO. The enigmatic epidemiology of nasopharyngeal carcinoma. Cancer Epidemiol Biomarkers Prev. 2006;15:1765-77.

[2] Wei WI, Sham JS. Nasopharyngeal carcinoma. Lancet. 2005;365:2041-54.

[3] Leung TW, Tung SY, Sze WK, Wong FC, Yuen KK, Lui CM, et al. Treatment results of 1070 patients with nasopharyngeal carcinoma: an analysis of survival and failure patterns. Head Neck. 2005;27:555-65.

[4] Jiang L, Wang P, Chen H. Overexpression of FOXM1 is associated with metastases of nasopharyngeal carcinoma. Ups J Med Sci. 2014;119:324-32.

[5] Nicholls JM, Agathanggelou A, Fung K, Zeng X, Niedobitek G. The association of squamous cell carcinomas of the nasopharynx with Epstein-Barr virus shows geographical variation reminiscent of Burkitt's lymphoma. J Pathol. 1997;183:164-8.

[6] Baujat B, Audry H, Bourhis J, Chan AT, Onat H, Chua DT, et al. Chemotherapy in locally advanced nasopharyngeal carcinoma: an individual patient data meta-analysis of eight randomized trials and 1753 patients. Int J Radiat Oncol Biol Phys. 2006;64:47-56.

[7] Chua DT, Ma J, Sham JS, Mai HQ, Choy DT, Hong MH, et al. Long-term survival after cisplatin-based induction chemotherapy and radiotherapy for nasopharyngeal carcinoma: a pooled data analysis of two phase III trials. J Clin Oncol. 2005;23:1118-24.

[8] Lun SW, Cheung ST, Lo KW. Cancer stem-like cells in Epstein-Barr virus-associated nasopharyngeal carcinoma. Chin J Cancer. 2014;33:529-38.

[9] Takeishi S, Nakayama KI. To wake up cancer stem cells, or to let them sleep, that is the question. Cancer Sci. 2016;107:875-81.

[10] Magee JA, Piskounova E, Morrison SJ. Cancer stem cells: impact, heterogeneity, and uncertainty. Cancer Cell. 2012;21:283-96.

[11] Chen K, Huang YH, Chen JL. Understanding and targeting cancer stem cells: therapeutic implications and challenges. Acta Pharmacol Sin. 2013;34:732-40.

[12] Abdal Dayem A, Choi HY, Yang GM, Kim K, Saha SK, Cho SG. The Anti-Cancer Effect of Polyphenols against Breast Cancer and Cancer Stem Cells: Molecular Mechanisms. Nutrients. 2016;8.

[13] Dandawate PR, Subramaniam D, Jensen RA, Anant S. Targeting cancer stem cells and signaling pathways by phytochemicals: Novel approach for breast cancer therapy. Semin Cancer Biol. 2016;40-41:192-208.

[14] Yang T, Liu J, Luo F, Lin Q, Rosol TJ, Deng X. Anticancer properties of Monascus metabolites. Anticancer Drugs. 2014;25:735-44.

[15] Gunde-Cimerman N, Cimerman A. Pleurotus fruiting bodies contain the inhibitor of 3-hydroxy-3-methylglutaryl-coenzyme A reductase-lovastatin. Exp Mycol. 1995;19:1-6.

[16] Ruano G, Seip R, Windemuth A, Wu AH, Thompson PD. Laboratory Medicine in the Clinical Decision Support for Treatment of Hypercholesterolemia: Pharmacogenetics of Statins. Clin Lab Med. 2016;36:473-91.

[17] Yang T, Yao H, He G, Song L, Liu N, Wang Y, et al. Effects of Lovastatin on MDA-MB-231 Breast Cancer Cells: An Antibody Microarray Analysis. J Cancer. 2016;7:192-9.

[18] Afzali M, Vatankhah M, Ostad SN. Investigation of simvastatin-induced apoptosis and cell cycle arrest in cancer stem cells of MCF-7. J Cancer Res Ther. 2016;12:725-30

[19] Parikh A, Childress C, Deitrick K, Lin Q, Rukstalis D, Yang W. Statin-induced autophagy by inhibition of geranylgeranyl biosynthesis in prostate cancer PC3 cells. Prostate. 2010;70:971-81.

[20] Ogunwobi OO, Beales IL. Statins inhibit proliferation and induce apoptosis in Barrett's esophageal adenocarcinoma cells. Am J Gastroenterol. 2008;103:825-37.

[21] Liu H, Liang SL, Kumar S, Weyman CM, Liu W, Zhou A. Statins induce apoptosis in ovarian cancer cells through activation of JNK and enhancement of Bim expression. Cancer Chemother Pharmacol. 2009;63:997-1005.
[22] Zhen Y, Ye Y, Yu X, Mai C, Zhou Y, Chen Y, et al. Reduced CTGF expression promotes cell growth, migration, and invasion in nasopharyngeal carcinoma. PLoS One. 2014;8:e64976.

[23] Jia W, Ren C, Wang L, Zhu B, Gao M, Zeng F, et al. CD109 is identified as a potential nasopharyngeal carcinoma biomarker using aptamer selected by cell-SELEX. Oncotarget. 2016.

[24] Ren KQ Cao XZ, Liu ZH, Guo H, Quan MF, Liu F, et al. 8-bromo-5-hydroxy-7-methoxychrysin targeting for inhibition of the properties of liver cancer stem cells by modulation of Twist signaling. Int J Oncol. 2013;43:1719-29.

[25] Liu Y, Chen C, Qian P, Lu X, Sun B, Zhang X, et al. Gd-metallofullerenol nanomaterial as non-toxic breast cancer stem cell-specific inhibitor. Nat Commun. 2015;6:5988.

[26] Bhattacharyya J, Bellucci JJ, Weitzhandler I, McDaniel JR, Spasojevic I, Li X, et al. A paclitaxel-loaded recombinant polypeptide nanoparticle outperforms Abraxane in multiple murine cancer models. Nat Commun. 2015;6:7939.

[27] Li H, He G, Yao H, Song L, Zeng L, Peng X, et al. TGF-beta Induces Degradation of PTHrP Through Ubiquitin-Proteasome System in Hepatocellular Carcinoma. J Cancer. 2015;6:511-8.

[28] Chou TC, Talalay P. Quantitative analysis of dose-effect relationships: the combined effects of multiple drugs or enzyme inhibitors. Adv Enzyme Regul. 1984;22:27-55.

[29] Song LB, Yan J, Jian SW, Zhang L, Li MZ, Li D, et al. [Molecular mechanisms of tumorgenesis and metastasis in nasopharyngeal carcinoma cell sublines]. Ai Zheng. 2002;21:158-62.

[30] Gauthaman K, Fong CY, Bongso A. Statins, stem cells, and cancer. J Cell Biochem. 2009;106:975-83.

[31] Speck NA, Stacy T, Wang Q, North T, Gu TL, Miller J, et al. Core-binding factor: a central player in hematopoiesis and leukemia. Cancer Res. 1999;59:1789s-93s.

[32] Chanmee T, Ontong P, Kimata K, Itano N. Key Roles of Hyaluronan and Its CD44 Receptor in the Stemness and Survival of Cancer Stem Cells. Front Oncol. 2015;5:180.

[33] Zhang Q, Feng Y, Kennedy D. Multidrug-resistant cancer cells and cancer stem cells hijack cellular systems to circumvent systemic therapies, can natural products reverse this? Cell Mol Life Sci. 2016.

[34] Al-Hajj M, Wicha MS, Benito-Hernandez A, Morrison SJ, Clarke MF. Prospective identification of tumorigenic breast cancer cells. Proc Natl Acad Sci U S A. 2003;100:3983-8.

[35] Dolfi SC, Chan LL, Qiu J, Tedeschi PM, Bertino JR, Hirshfield KM, et al. The metabolic demands of cancer cells are coupled to their size and protein synthesis rates. Cancer Metab. 2013;1:20.

[36] Thibault A, Samid D, Tompkins AC, Figg WD, Cooper MR, Hohl RJ, et al. Phase I study of lovastatin, an inhibitor of the mevalonate pathway, in patients with cancer. Clin Cancer Res. 1996;2:483-91.

[37] Rossi A, Molinari R, Boracchi P, Del Vecchio M, Marubini E, Nava M, et al. Adjuvant chemotherapy with vincristine, cyclophosphamide, and doxorubicin after radiotherapy in local-regional nasopharyngeal cancer: results of a 4-year multicenter randomized study. J Clin Oncol. 1988;6:1401-10.

[38] Ahlmann M, Hempel G. The effect of cyclophosphamide on the immune system: implications for clinical cancer therapy. Cancer Chemother Pharmacol. 2016;78:661-71.

[39] Agostinis P, Berg K, Cengel KA, Foster TH, Girotti AW, Gollnick SO, et al. Photodynamic therapy of cancer: an update. CA Cancer J Clin. 2011;61:250-81.

[40] Spring BQ, Rizvi I, Xu N, Hasan T. The role of photodynamic therapy in overcoming cancer drug resistance. Photochem Photobiol Sci. 2015;14:1476-91.

[41] Lin L, Xiong L, Wen Y, Lei S, Deng X, Liu Z, et al. Active Targeting of Nano-Photosensitizer Delivery Systems for Photodynamic Therapy of Cancer Stem Cells. J Biomed Nanotechnol. 2015;11:531-54.

[42] van Marion DM, Domanska UM, Timmer-Bosscha H, Walenkamp AM. Studying cancer metastasis: Existing models, challenges and future perspectives. Crit Rev Oncol Hematol. 2016;97:107-17.

[43] Chan KK, Oza AM, Siu LL. The statins as anticancer agents. Clin Cancer Res. 2003;9:10-9 